\title{
A Preliminary Assessment for Inshore Fishing UAV (ISFUAV)
}

\author{
Zacharie Mbaitiga ${ }^{\mathrm{a}}$, Kyuhei Honda ${ }^{\mathrm{b}, *}$ \\ aNational Institute of Technology, Okinawa College, 905 Henoko, Nago, Okinawa, 905-2192,Japan \\ ${ }^{b}$ National Institute of Technology, Oita College, 1666 Maki, Oita, 870-0152, Japan \\ *Corresponding Author: honda@oita-ct.ac.jp
}

\begin{abstract}
This paper presents an assessment of what is needed to develop the inshore fishing UAV (ISFUAV) capable of flying from the seashore over the sea for a distance of 500 meters to one kilometer before the on-board gripper releases the fish food attached to the fish hooks for catching fish. The duration of the aircraft flight will last for about 20 minutes minimum. The aircraft will be equipped with many sensors for safe navigation including vision system.
\end{abstract}

Keywords: UAV, Fishing drone, Seashore, Over the sea

\section{Introduction}

With the ongoing progress in technology in general and computer science in particular, the Unmanned Aerial Vehicles in short term UAVs are becoming a promising research area that could lead over the robotics and avionics, and others research fields in the years to come. This is due to the current research topics on the UAVs around the planet. At the beginning the UAVs were accepted very slowly by the military in 1990 's, but when the time goes by they brought a real revolution in military operations. These military operations include:

\section{A. Non-offensive operations such as:}

- Day and night reconnaissance and surveillance.

- A collection of information to support political decision making in crisis management.

- Battle damage assessment description of operations.

- Nuclear, biological and chemical detection.

- Communication and non-communication jamming.

B. Offensive operations such as:

- Target acquisition (the process of detecting, identifying, and locating a target in sufficient detail to permit the effective employment of weapons).
- Target designation (the process of marking or other-wise pointing to a target by any means).

- Close air support and defense against theatre ballistic missile and cruise missile.

- Mobile target strikes.

Presently, the UAVs are considered not only as a standard tool in military thinking ${ }^{(1)}$ but also they have reached a high level of reliability with a large variety of civil applications. These civil applications include:

- $\quad$ River and sea application monitoring.

- River and sea pollution monitoring.

- Detection of pollutants in the atmosphere.

- Assistance in the case of criminal actions.

- Video transmission of sports competition and so on.

Due to the impact of the UAVs on every part of our living style, academic institutions, research laboratories and agencies have been recently shifting their research interests from robotics to UAVs for both educational purposes and commercial. Among those institutions we can mention the University of Minnesota with UAV laboratory's aim to conduct flight research on navigation systems, guidance and control systems to enable future commercial aircraft and small UAVs that are significantly safer and more fuel efficient $^{(2)}$. This UAV laboratory also supports education which brings real world aircraft development and flight testing experience into undergraduate and graduate courses.

The University of Florida does not miss the party. It disposes an Unmanned Aircraft System Research Program in short UFUSRP that focuses on the development of small unmanned aircraft systems that are both affordable and addresses scientific questions in natural resources through remote sensing ${ }^{(3)}$. Henri Eisenbeiss et al. proposed the UAV systems and their applications for the photogrammetric recording and documentation of cultural heritage ${ }^{(4)}$. Serge Wich et al. proposed a preliminary assessment of conservation drone for Sumatran orangutan distribution and density. The assessment focuses on whether or not they 
could detect nests of the critically endangered Sumatran orangutans on imagery acquired from a camera mounted drone to determine distribution and density ${ }^{(5)}$. Jones GPN et al. proposed an assessment of small unmanned aerial vehicles for wildlife research where they captured high-quality progressive scan video of a number of landscapes and wildlife species ${ }^{(6)}$. Unfortunately, the UAV system was unable to collect geo-reference imagery and at the same time it was difficult to deploy it in unimproved areas. But the performance of the autonomous control systems and the quality of the progressive-scan imagery indicated strong promise for the future UAVs as useful field tools. C. Goerzen and Kong B. Mettler did a survey of motion planning algorithm from the perspective of autonomous UAV guidance ${ }^{(7)}$ with aim to provide an overview of existing motion planning algorithm while adding perspective and practical examples from UAV guidance approaches. Getins, Weigank and Shoning contributed to the UAV applications by assessing biodiversity in forest using high resolution images and unmanned aerial vehicles ${ }^{(8)}$. All these surveys and assessments have shown how the UAVs are showing signs of future success in many different areas that will change our life style although the research on the UAVs are on their early stage. They will without doubt contribute to sustain our society as well. The purpose of this work is to make an assessment on how to develop a robust and affordable inshore fishing UAV in order to help fisherman or fishing lovers in all level that do not have a boat and could not go far away in the sea from the seashore to enjoy fishing during their spare time.

\section{Overview of Civilian UAV Applications}

Unmanned Aerial Vehicles (UAVs) have been a hot button issue as of late. Some in the near future will show their ability to perform many tasks humans cannot be able to do. Others will offer the opportunity for entertainment such as playing with kids, wife, friends and family members. In fact, the first civilian science-related mission involved counting sand hill cranes. A task that usually involves a biologist flying in a plane or helicopter but with the use of UAVs which have the ability to fly very close without scaring off animals, scientists were able to utilize a thermal imagery camera on the UAVs called Raven to count the cranes while they were settled in the wetland during the evening. The following are some civilian UAV applications.

\section{A. UAV guiding students around the campus}

The Massachusetts Institute of Technology (MIT) has developed an unmanned autonomous vehicle system which uses GPS and cameras to guide students and visitors around the campus. The system called Skycall allows its users to call it via phone call and then flies to their location to guide them on to their destination. The prototyped Skycall system consists of a quad-copter drone equipped with on board autopilot, cameras, Wi-Fi, GPS navigation and sensors that allow it fly autonomously to specific locations. Not only does Skycall copter have an onboard camera that provides information to the base location upon a user but it also has a manually controlled camera which is accessible to users via the Skycall application. The Skycall application enables users to make requests and the UAV to both locate and wirelessly communication with them. It also detects if a user is falling behind and ask them to close the distance.

\section{B. Amazon UAV delivery service}

Operationally or technically the Amazon UAV Delivery Service has not happened yet, but it is still something that will draw our attention on how the UAVs applications continue to develop in the coming years. The Amazon UAV Delivery Service prototype tested and shown on the TV is to deliver a package under five pounds $(23 \mathrm{~kg})$ to a costumer within a 10 mile $(16 \mathrm{~km})$ radius of an Amazon fulfillment center. The UAV will drop off the package right at the costumer doorsteps. This is a matter of time but far this to be successful, a certain number of issues are needed to be addressed first.

\section{Drug bust using UAV by USA Coast Guard}

In July 2003, the USA coast guard used a UAV that they developed to aid in the interdiction of 600 kilograms of cocaine. This is the first time the coast guard used a UAV in an interdiction operation. The UAV called Scan-Eagle can monitor a suspected go-fast vessel for more than 90 hours. When the UAV located the suspected vessel, it maintained constant on-scene surveillance until the short range recovery helicopter and over the horizon cutter boats arrived to interdict and apprehend the vessels crew. The UAV has an integrated EO900 imager electro-optical model combining an electro-optical telescope with Insitu's electro-optical camera which provides $170 \mathrm{x}$ zoom. It is gyro-stabilized with coordinate hold mode and includes a video progressive board, picture-in-picture display, and articulated nose enclosure. The coast guard has identified a cutter-based UAV as a key component to enhance the 
operation effectiveness of its major cutter fleet through on demand, persistent surveillance capabilities. So with the ongoing forward of the UAVs civil applications, what type of impact can we expect when it comes to using the UAVs in the scientific research community in the years to come? Perhaps in an immediate one as most developed drones are currently under experimentations for safety concerned by federal movement, research agencies or academic institutions. Based on the reported UAVs and civil aircraft incident in the air, the Federal Aviation Administration of some countries are working on new guidelines, which would allow for the full integration of private commercial UAVs into airspace. Some company leaders in the scientific community are already waiting eagerly for the new rules to be adopted as they expect the approval to result in safer, lower-cost data capture and so forth. This approval will pave the way for the use of the current develop shore fishing UAV.

\section{Fishing UAV Specifications}

This project when completed, the Inshore Fishing UAV will operate in the sea level for a duration of more than 20 minutes. The distance range will be between 500 meters to one kilometer for about 30 meters high above the sea. The UAV will carry the fish food attached to the fishing hooks. Such a UAV should have specifications that fit the project aim. To this end, a survey is conducted on the UAVs community where the Phantom 3 has been selected. The phantom 3 is a quadrotor with built-in flight control system with integrated gimbal and camera as shown in Fig.1.

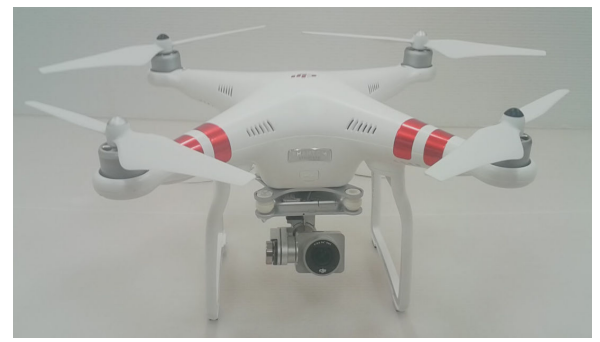

Fig.1. Phantom 3 (prospective Inshore Fishing UAV).

The Phantom3 features a flight control assistance (FCA) port, a camera port and specialized battery compartment for its flight battery. The flight control system can communicate with PC assistance through a micro-USB cable between the phantom assistant (FCA) port and the PC. This features make it possible for the user to use assistance to configure the aircraft and upgrade the phantom firmware. One of the strongest points of the selected drone is the antenna orientation that can be kept and pointing skyward to the ground for maximum control range during the flight. The phantom 3 is also equipped with a camera having a resolution of $4000 \times 3000$, sensor with size of $1 / 2.3$ ". The camera supports burst shots, continuous capture and time capture, and exports to both Adobe DNG Raw and JPEG. For aerial video, it can shoot in ultra HD and can even shoot for internet ready movies. The LED light indicators are also attached to the Inshore Fishing Aerial Unmanned

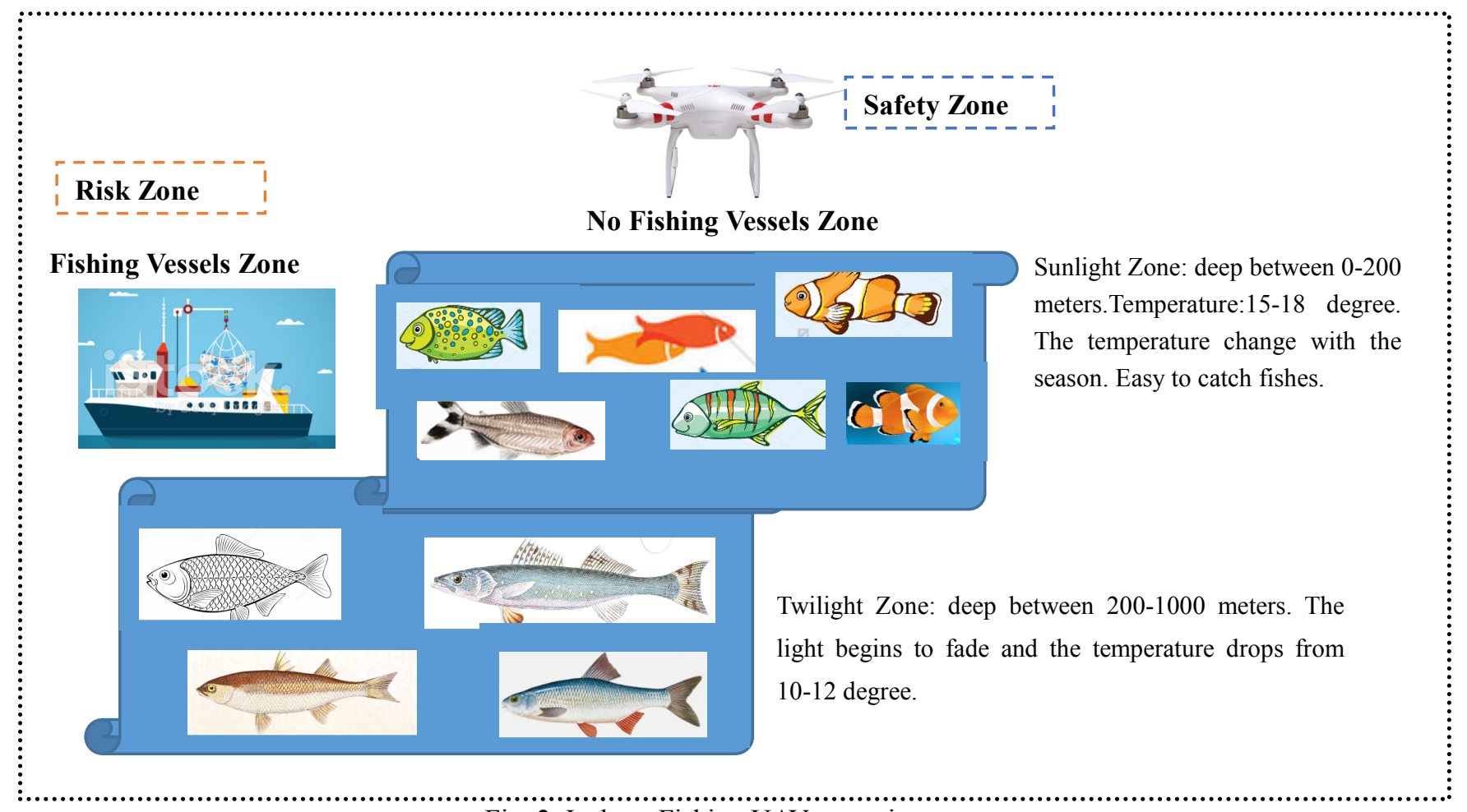

Fig. 2. Inshore Fishing UAV operating zone. 
Vehicle (ISFUAV). The LEDs light are installed at the front and the rear of the ISFUAV. The front LEDs are used for indicating where the nose of the Aerial Unmanned Fishing Vehicle is during its operations. They can light up solid red after the motors have started spinning. While the rear LEDs light indicators light up to show the aircraft current flight status once the light battery is also powered on. The 3-axial stabilizer Gimbal is also attached to the aircraft with the following two working modes. The non-FPV mode and FPV mode. The FPV is defined as "First Person View".

Table 1. Aircraft Specifications.

\begin{tabular}{|l|l|}
\hline Battery & DJI 4480mAh Li-Po battery \\
\hline Weight & $1216 \mathrm{~g}$ \\
\hline Max Ascent/Descent Speed & Ascent: $5 \mathrm{~m} / \mathrm{s}$, Descent: $3 \mathrm{~m} / \mathrm{s}$ \\
\hline Max Flight Speed & $16 \mathrm{~m} / \mathrm{s}$ \\
\hline Motor Diagonal Length & $350 \mathrm{~mm}$ \\
\hline
\end{tabular}

\section{Methodology}

We are living in a world surrounded by sea extended from the Pacific Ocean to the Mediterranean by passing to the Red Sea in Egypt which mark the history of humanity with its all kinds of wealth. When we compare the sea to the land with its millions kinds of animals and all living creatures, the sea also has an uncounted number of fishes and living species that we enjoy every day. But when we take a few times to think about where these fishes and species we are buying every day at the marketplace come from? The answer is: these fishes and species mostly come from the sea and usually those people who possess either a small or big boat have the privilege to go far away deep into the sea for about a mile or more where they can catch a variety of fishes such as tuna, mackerel, salmon and so on. While those who do not have a boat can just struggle around the shore with their small hooks and can stay for an hour sometimes three to four hours without catching even small fishes. Even if the opportunity has been given to them to catch fish, what they can catch are only tiny fishes. So in order to take the advantage of the technology and to give an opportunity to whoever wants to enjoy fishing, we came up with the idea to develop the Inshore Fishing Unmanned Aerial Vehicle (ISFUAV). The ISFUAV operation will be limited on to inshore fishing and not offshore. The biggest difference between inshore and offshore fishing is the depth of the water. Inshore fishing is any fishing that takes place in the water up to 30 meters deep. Since the waters are calm, vessels are less intense and a boat can even not be seen, and there more fishes are playing around. With 30 meters deep of water is an acceptable line-in-the-sand acting as the border between the two shores (inshore and offshore). When we are fishing inshore, we are usually within a few miles of shore. Once we hit over 30 meters deep, we are offshore the deep fishing zone. Here the boat becomes more robust, the fishing becomes more athletic and less of relaxing day out on the boat and more often than not within the casting distance of the working popular spots. Our developed ISFUAV can extend the fishing depth from 30 meters to 80 meters and fly over the sea for a distance of 500 meters and 30 meters high above the sea level as shown in Fig.2. The flight duration will be at least 20 minutes.

As can be seen in Fig.2, small and medium fishes such as trout and bass often live near the surface of the water looking for food and attempt to escape the jaws of larger fish. This environment fits well the ISFUAV operation area and the fishes can be easy catch. The ISFUAV will be equipped with a fish detection sensor if possible. This sensor will be used to locate the fish under the sea from 30 meters to 50 meters deep depending on the sensor performance. This sensor is under investigation and will be decided very shortly when the project development starts. In case the fish detection sensor cannot be integrated into the ISFUAV due to some constraints such as the load of the sensor, another appropriate sensor that fits the project will be investigated. Under the UAV a small gripper will be integrated that will serve as the fish food and the fish hooks carrier. The UAV will have two control system modes. The first control system will focus on flying navigation based remote control in order to check the performance of all systems including the duration of the aircraft, battery life span and to make sure that all different navigation function work well. The second control system will be to make the aircraft to fly automatically based on the flight distance from the seashore up to 500 meters over the sea level with a speed of $10-12 \mathrm{~m} / \mathrm{s}$. The aircraft will also be integrated with a vision system for video recording during the flight operation (Table 2).

Table 2. Camera Specifications.

\begin{tabular}{|l|l|}
\hline Operating Environment & $0^{\circ} \mathrm{C}-40^{\circ} \mathrm{C}$ \\
\hline Sensor Size & $1 / 2.3 "$ \\
\hline Effective Pixel & $12 \mathrm{Mega}$ Pixels \\
\hline Resolution & $4000 \times 3000$ \\
\hline HD Recording & $1520 \mathrm{p} 30,720 \mathrm{p} 60$ \\
\hline Recording FOV & 94 deg. \\
\hline
\end{tabular}


From this camera we will record videos, burst shots and continuous capture and timed capture. In order to avoid a sudden crash due to battery failure, the ISFUAV is equipped with a 3-axis stabilization Gimbal as described in Table III. This vision system is connected to a PC using a Micro-USB cable to copy image files from the vision system to the PC.

Table 3. 3-axis Stabilization Gimbal Specifications.

\begin{tabular}{|l|l|}
\hline Stabilization & 3-axis(pitch, roll, yaw) \\
\hline Controllable Range & Pitch:-90deg. to +30deg. \\
\hline
\end{tabular}

This gimbal has two working modes. Non-FPV (First person view) mode and FPV mode. In Non-FPV mode, the gimbal will stabilize across 3-axial for smooth aerial creativity, while in FPV mode, the gimbal will lock the movements of the aircraft for a FPV experience. The system has a function to self-check each time when the flight battery is on and alert the base station in case of low power so that the aircraft operator can take preventive action such as calling back the aircraft to the base station before it can crash.

\section{Expected Results and Discussion}

The ISFUAV will operate in a zone where there will be not any obstacle within a perimeter of 500 meters by 500 meters. From the base station at the seashore, the equipped camera will be activated to live stream to send data obtained to the base station in a real time in order to check the aircraft location, and the surroundings before the activation of the gripper carrying the fish food attached to the fish hooks. After the hooks are released at the casting spot, the aircraft should return to the base station to carry out a second attempt and so on. The distance from the first released hooks and the second is to be set from 50 meters to 100 meters in width. After a fish is caught on the hook, the fisherman or the assistant pulls the fishing line and pulls out the fish from the water at the seashore. The top view of the ISFUAV operating zone is shown in Fig. 3.

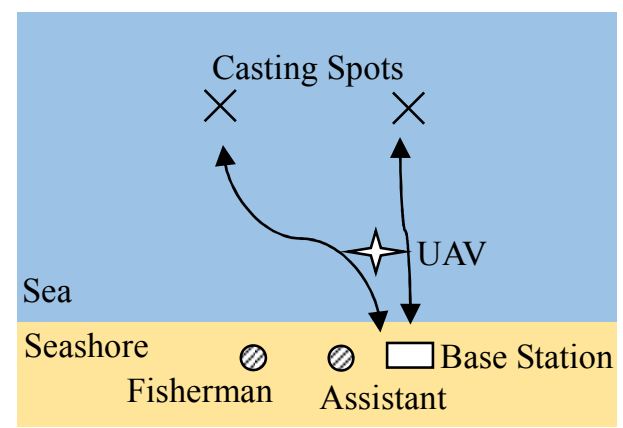

Fig.3. Top view of the ISFUAV casting zone.
In order to examine the ISFUAV loading capacity, so that we can adjust the weight of the gripper with its holder and fishing tackle such as fishing hooks, sinkers and floats, we conducted a preliminary experiment using a Phantom 3 Standard UAV with manual remote control. As shown in Fig. 4, we used two plastic bottles filled with water while the total weight changed from about 400 grams to 1 kilogram in steps of 100 grams. The UAV can lift up the bottles when they weigh up to 900 grams. However, we sometimes observed intermittent lack of climbing power to keep the altitude during its flight when they weighed over 600 grams. The lack of climbing power observed during this preliminary test gives us an indication that it is necessary to convey a command of motion in a skyward direction when the load is heavy and it is better to load less than 600 grams in total. At present, we are planning to design the gripper including its electronic circuits and its batteries which weighs less than 200 grams so that we can load up to 400 grams of fishing tackle including fishing baits.

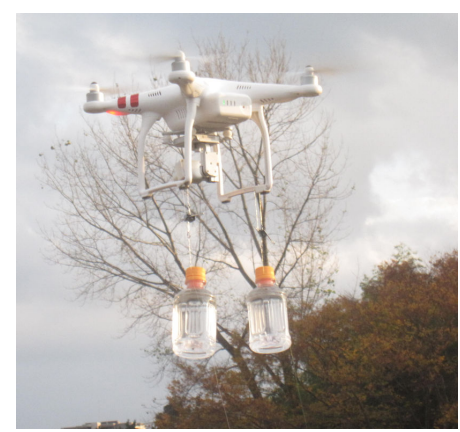

Fig.4. Experimental flight with two weights.

We have built a prototype set of electronic circuits using $2.4 \mathrm{GHz}$ wireless modules to remotely control a motor for the gripper and conducted an experiment to activate the motor in the school playground. We confirmed that the remote control for the motor succeeded at a distance of about 235 meters, although the size of the playground prevented us from conducting more distant experiments. We need to find a more spacious place to verify its catalog spec of more distant communication capability.

For more effective carriage of the hooks, we conducted an experiment where we connected two fishing lines to the UAV. The UAV can fly without tangling both fishing lines for a distance of more than 180 meters, which gives us an indication that the UAV can visit two casting spots in only one flight attempt from the seashore.

Note that these preliminary tests are not the fishing project data yet, but are needed to better design the ISFUAV project. 
We expect to release at least for the time being 5 to 10 hooks with 30-minute time frame. Once the system is tested successful then we will move on to develop the commercial ISFUAV that can be available to anyone who wishes to enjoy fishing where the assistant fisherman will not be necessary anymore.

The ISFUAV itself will do everything automatically. Although we intend to operate our ISFUAV in a free obstacle zone, we cannot tell for sure due to the number of small drones nearby so its path planning and collision avoidance will be in schedule for implementation in order to deal with the sudden popping-up of drones, birds or anything in between as the environment is not always static. That is to say or in other word it can be changed with many uncertain factors. In the air there are not only a number of static threats which have been known a priori, but also caused by pop-up on some threats that are known only when other UAVs or birds maneuver into their proximity ${ }^{(9)}$. Furthermore, even those static threats locations have been detected ahead of time, their threat grade or threat scope may be changing frequently, which also makes them uncertain. Considering these uncertain factors, the preplanned trajectories often are not adapted to the practice under the changing environment in the air operation.

In order to minimize the ISFUAV sudden crash or to avoid a sudden collision and to increase the probability chance of the ISFUAV survival, the path planning is essential $^{(1,9)}$. Suppose maximizing the probability of the ISFUAV mission in a dynamic and uncertain environment will succeed, it is desirable to assign the ISFUAV to conduct where some of minimum criteria are implemented into the navigation system of the aircraft ${ }^{(10)}$. Thus, the problem of the ISFUAV coordinate path planning in a dynamic and uncertain environment is put ahead.

Path Planting: The path planning will concern both the Go-For Mission (GFM) and Return to the Base Station (RBS) after completing its fishing mission. The GFM and RBS will require determining an estimated time of arrival (ETA) at the specific destination spot. The ISFUAV should select its path and adjust its flight velocity corresponding to the ETA. That is to say that the aircraft trajectory and velocity will change coordinately when the sudden popping-up of threat occur. The change of the ISFUAV trajectory and velocity will be influenced by the help of data coming in a real time from the sensors equipped to the aircraft.
Collision Avoidance: The collision avoidance will require that the trajectory of the ISFUAV and sudden pop-up of unknown flight aircraft should have no overlaps or cross each other. All sensors on the flying aircraft will be programmed in the way that the distance between the sudden pop-up unknown flight aircraft and the ISFUAV are to be at least 50 to 100 meters wide by base on the information from sensors.

\section{Conclusion}

This paper described a preliminary assessment of information needed to develop an inshore fishing unmanned aerial vehicle. The aim of this project is to help all fisherman at all lever including wheelchair people to enjoy fishing from the seashore during their spare time. The project is intended to be completed as soon as possible and make on the market for commercial.

\section{References}

(1) H. Duan, and P. L: "Bio-inspired Computation in Unmanned Aerial Vehicles", Springer ISBN: 978-3-642-41195-3

(2) http://www.uav.aem.umn.edu/

(3) http://uav.ifas.ufl.edu/people.shtml

(4) Eisenbeiss, H. : "A mini unmanned aerial vehicle (UAV): System overview and image acquisition", International workshop on processing and visualization using high resolution imagery, Vol. XXXVI-5/W1, 2004

(5) Serge Wich et al. : "A Preliminary Assessment of Using Conservation Drones for Sumatran Orang-utan (Pongo abelii) Distribution and Density",Journal of Unmanned Vehicle Systems, 2016, 4(1): 45-52

(6) George Pierce Jones, IV, Leonard G. Pearlstine and H. Franklin Percival: " An Assessment of Small Unmanned Aerial Vehicles for Wildlife Research", Wildlife Society Bulletin, vol. 34, No.3,p., 2016

(7) C. Goerzen, Z.Kong, B.Mettler: "A Survey of Motion Planning Algorithms from the Perspective of Autonomous UAV Guidance",Journal of Intelligent and Robotic Systems 57(1-4):65-100, January 2010

(8) Stephan Getzin, Kerstin Wiegand and Ingo Schoning: "Assessing biodiversity in forests using very highresolution images and unmanned aerial vehicles", Journal of Methods in Ecology and Evolution 2012, 3, 397-404

(9) M. Zucker, J. Kuffner, M. Branicky: "Multipartite RTTs for rapid replanning in dynamic environments", Proc. of IEEE International Conference on Robotics and Automation, 2007

(10) R. Beard, T. McLain: "Multiple UAV cooperative search under collision avoidance and limited range communication constraints",Proc. of IEEE Int. Conf. on Decision and Control,pp.25-30, 2003 